\title{
The management of tumor lysis syndrome
}

\author{
Elvira Rampello, Tiziana Fricia and Mariano Malaguarnera*
}

\section{SUMMARY}

The manifestation of tumor lysis syndrome (TLS) occurs when the destruction of tumor cells releases breakdown products that overwhelm the excretory mechanisms of the body. A cardinal sign is hyperuricemia, leading to uric acid nephropathy. Other signs are hyperkalemia, hyperphosphatemia and secondary hypocalcemia. Conventional management of TLS consists of aggressive intravenous hydration, diuretic therapy, urinary alkalization, and inhibition of urate production by high-dose allopurinol. Urate oxidase has been used in the management of patients at risk for TLS and recently the recombinant urate oxidase rasburicase was developed. Several data indicate that rasburicase is effective and well tolerated in the prevention and treatment of chemotherapy-induced hyperuricemia. Treatment options of hyperkalemia include sodium polystyrene sulfonate, hypertonic glucose and insulin, loop diuretics, and bicarbonate. Treatment of hyperphosphatemia reduces dietary phosphate intake and includes phosphate binders such as aluminum hydroxide and aluminum carbonate. When recurrent hypocalcemia is present, a continuous intravenous infusion of calcium gluconate can be initiated. Hemodialysis should be considered for every patient with excessively elevated uric acid, phosphate and/or potassium and in those patients with acute renal failure to control urinary volume and manage uremia.

KEYWORDS: acute renal failure, hyperkalemia, hyperuricemia, hypocalcemia, tumor lysis syndrome

\section{REVIEW CRITERIA}

The information for this review was obtained by searching the PubMed and MEDLINE databases for articles published up until 30 December 2005. Electronic early-release publications were also included. Only articles published in English were considered. The search terms used included "hyperuricaemia" in association with other search terms: "tumor lysis syndrome", "hyperkalemia", "hyperphosphatemia", "hypocalcemia", "acute renal failure", "allopurinol", "urate oxidase", and "rasburicase". When possible, primary sources have been quoted. Full articles were obtained and references were checked for additional material when appropriate.

\section{E Rampello and T Fricia are medical doctors, and M Malaguarnera is Associate Professor of Oncology, all at the Department of Senescence, Urological and Neurological Sciences, Catania University, Catania, Italy.}

\section{Correspondence}

*Department of Senescence, Urological and Neurological Sciences, University of Catania, Ospedale Cannizzaro, Via Messina 829, I-95126 Catania, Italy malaguar@unict.it

Received 15 March 2005 Accepted 3 April 2006

www.nature.com/clinicalpractice

doi:10.1038/ncponc0581

\section{INTRODUCTION}

Tumor lysis syndrome (TLS) is characterized by rapid and massive destruction of tumor cells, which leads to a subsequent release of cellular breakdown products sufficient to overwhelm the excretory mechanism and the body's normal functional capacity. This condition can result in severe impairment of various organs, including the heart, kidney, muscle and nervous system. ${ }^{1}$ The cardinal signs of TLS are hyperuricemia, leading to uric acid nephropathy, hyperphosphatemia and secondary hypocalcemia and hyperkalemia. TLS often manifests itself as a direct result of chemotherapy, radiotherapy or immunotherapy. ${ }^{2,3}$ Some correlations between more-aggressive cytotoxic treatment and TLS have been reported, but these correlations probably reflect the biology of the disease rather than the treatment. Moreover, the syndrome can occur spontaneously in the absence of treatment, which contributes to a worse clinical outcome because there is no prophylaxis when TLS occurrence is spontaneous. ${ }^{4-7}$

Since 1870, various metabolic disturbances have been recognized in association with cancer therapy. ${ }^{8}$ In 1929 it was reported that, in patients with chronic leukemia, irradiation of bulky disease resulted in a marked increase in uric acid and an associated decrease in tumor mass. ${ }^{9}$ In 1977 , a patient affected by advanced gastrointestinal carcinoma was the first reported case of the spontaneous form of the syndrome associated with acute renal failure (ARF) due to uric acid nephropathy. ${ }^{10}$ TLS is most often associated with the treatment of Burkitt's lymphoma, acute lymphoblastic leukemia and other high-grade lymphomas, but can also be associated with chronic leukemias and low-grade lymphomas. ${ }^{11-17}$ TLS has also been reported in patients with metastatic breast carcinoma, ${ }^{18-20}$ small-cell lung carcinoma, ${ }^{21-23}$ non-small-cell lung carcinoma, ${ }^{6,24}$ seminoma, ${ }^{25}$ thymoma, ${ }^{26}$ medulloblastoma, ${ }^{27}$ ovarian carcinoma, ${ }^{28}$ rhabdomyosarcoma, ${ }^{29}$ melanoma, ${ }^{1}$ vulvar carcinoma ${ }^{30}$ and Merkel-cell carcinoma (Box 1). ${ }^{31}$ TLS onset is more frequent in hematologic malignancies than 
Box 1 Symptoms and types of cancer associated with tumor lysis syndrome.

\section{Merkel-cell carcinoma}

A rare but highly malignant type of skin cancer usually found on sun-exposed body areas (e.g. head, neck, arms or legs) characterized by a firm, painless, shiny lump that can be red, pink, or blue in color and varies in size from 0.5 to $5 \mathrm{~cm}$ in diameter.

\section{Torsades des pointes}

A polymorphic ventricular tachycardia characterized by phasic changes in the amplitude and polarity of the ventricular complexes; often associated with a prolonged QT interval.

\section{Anion gap}

A measurement of the interval between the sum of 'routinely measured' cations and the sum of the 'routinely measured' anions in the blood; a high anion gap indicates metabolic acidosis.

\section{Thymoma}

A rare tumor of the thymus gland, which is located in the anterior mediastinum. The most common disease in people with thymoma is one in which the muscles are weak, called myasthenia gravis.

\section{Medulloblastoma}

Medulloblastoma is a highly malignant tumor representing the most common malignant posterior fossa tumor in the pediatric population. This tumor is characterized by the tendency to seed along the neuraxis, following cerebrospinal fluid pathways, and represents one of the few brain tumors to metastasize to extraneural tissues. Originally classified as a glioma, medulloblastoma is now referred to as a primitive neuroectodermal tumor.

\section{Rhabdomyosarcoma}

Rhabdomyosarcoma is a malignant soft tissue tumor found in children and it is believed to arise from a primitive muscle cell. The most common sites are the structures of the head and neck, the urogenital tract and the arms or legs.

in solid tumors because surgical intervention can reduce tumor load in the latter. TLS is more severe when there is a high tumor growth fraction and rapid tumor growth rates, and the syndrome is sensitive to chemotherapy.

\section{PATHOPHYSIOLOGY}

Cell destruction that occurs with aggressive treatment of malignant diseases causes a rise in excreted waste products and is accompanied by metabolic disturbance. Increased purine metabolism as a result of high tumor cell turnover leads to hyperuricemia. Cytolytic chemotherapy causes tumor cell breakdown and a rapid increase in plasma uric acid, potassium and phosphorus. These events together with hypocalcemia, which occurs as a consequence of increased phosphorus, are the major components of TLS. ${ }^{32}$ Development of acidosis can also be associated with TLS. Potassium, uric acid and phosphate are primarily excreted during renal clearance, and development of renal failure can further aggravate this electrolyte imbalance. ${ }^{33}$

Normally, the urate is filtered by the glomerulus, partial proximal tubular reabsorption and distal renal tubular secretion of the kidney. ${ }^{34}$ The urate sometimes crystallizes in the distal tubule, where it can reach high concentrations as a result of active secretion and ongoing tubular acidification. The rate of urate clearance is highly dependent on the glomerular filtrate flow rate and may fall significantly if dehydration is present. Dehydration can occur as a consequence of tumor development or treatment, resulting in ureteral obstruction and other complications that further compound dehydration. Urate nephropathy develops as a result of the acid conditions and urate crystal formation in the renal tubules and collecting ducts. This condition can cause complications leading to renal insufficiency and therapy-related death; acute urate nephropathy associated with TLS results from pathological urate crystal deposition in the distal renal tubules. In certain patients, renal tumors may coexist with kidney pathology induced by TLS, which can manifest before the initiation of chemotherapy or up to 5 days after the chemotherapy, especially with tumors that are highly proliferative and sensitive to chemotherapy. ${ }^{12}$

\section{CLINICAL FEATURES}

TLS should be suspected in patients with tumor burden who develop ARF and have hyperuricemia, hyperphosphatemia and elevated lactate dehydrogenase. The severity of the metabolic alterations (caused when uric acid levels are $>594.8 \mu \mathrm{mol} / 1$ [ $>10 \mathrm{mg} / \mathrm{dl}]$, serum potassium levels are $>6 \mathrm{mmol} / \mathrm{l}$, and serum phosphorus levels are $>1.62 \mathrm{mmol} / \mathrm{l}[>5 \mathrm{mg} / \mathrm{dl}]$ ) is influenced by the dose of chemotherapy, the size of tumor mass, the number of lysed cells and the patient's state of hydration and renal function. Specific clinical features of TLS are shown in Boxes 2 and 3.

\section{Hyperkalemia}

A rise in serum potassium levels occurs 6-72 hours after the initiation of chemotherapy. ${ }^{35}$ Symptoms 


Box 2 Risk factors associated with high risk
of tumor lysis syndrome.
Elevated LDH
Leukocytosis $\left(>50,000 \mathrm{~m}^{3}\right)$
Elevated uric acid and calcemia on admission
Elevated creatinine levels $(>141.4 \mu \mathrm{mol} / \mathrm{l}[>1.6 \mathrm{mg} / \mathrm{dl}])$
Decreased glomerular filtrate rate

Box 3 Predisposing factors for tumor lysis syndrome.

Tumor characteristics

High tumor growth fraction

Advanced stage of malignancy

Rapid tumor growth rate

Abdominal organ involvement

High sensitivity to chemotherapy

Patient characteristics

Compromised baseline renal function

Evidence of urinary tract obstruction via imaging

Polypharmacology

Drug-drug interactions

associated with hyperkalemia include lethargy, muscle weakness and paresthesia or paralysis, and electrocardiographic manifestations. The most common electrocardiogram abnormalities are peaked $T$ waves in the precordial leads, prolongation of the PR interval, flattening or absence of the $\mathrm{P}$ wave, widening of the $\mathrm{QRS}$ complex, and a sine wave' appearance, which is associated with severe hyperkalemia. ${ }^{36}$ Increased serum potassium levels can cause ventricular arrhythmias and lead to sudden death; low serum calcium and acidosis can exacerbate this predisposition (Table 1). Nonspecific symptoms, such as nausea and vomiting, can result from hyperphosphatemia, hypocalcemia, hyperuricemia and especially renal failure. These symptoms are often poorly managed with antiemetic treatments, which should be used with caution in the presence of renal or hepatic failure.

\section{Hyperphosphatemia and hypocalcemia}

Hyperphosphatemia develops 24-48 hours following initiation of chemotherapy, and can cause many kinds of symptoms, including decreased mental status, weakness, cramps, hyperreflexia, tetany and renal failure. Hyperphosphatemia can also cause dysrhythmias, including polymorphic ventricular tachycardia and torsades de pointes
(Box 1) due to a prolonged QT interval on the electrocardiogram. ${ }^{37}$ Physiologically, calcium influences neuromuscular excitability; therefore, hypocalcemia can cause muscle cramps, cardiac abnormalities, and pronounced blood coagulation deficiency (Table 1). ${ }^{38}$ Hypocalcemia is not usually symptomatic, but symptoms such as neuromuscular irritability and tetany, anorexia, vomiting, carpopedal cramps, seizures and spasms can develop. Hypocalcemia also causes dysrhythmias and cardiac arrest in extreme cases. $^{2,37}$ Hyperphosphatemia can also lead to calcium phosphate deposition in renal tubules.

\section{Hyperuricemia}

Hyperuricemia can develop within 48-72 hours of chemotherapy. Uric acid is an end product of purine metabolism and is excreted by the kidneys. Hyperuricemia can cause a myriad of abnormal physical symptoms, including nausea, vomiting, lethargy, oliguria, anuria, anorexia, and hematuria. Blood urea nitrogen and creatinine levels can rise, causing edema, acute acid nephropathy and renal failure (Table 1). ${ }^{39}$ Uric acid exists in a soluble ionized form at $\mathrm{pH}$ 7.0, but its solubility decreases markedly as the urinary $\mathrm{pH}$ decreases in the renal collecting ducts and deep cortical and medullary vessels, where the luminal $\mathrm{pH}$ approaches $\mathrm{pH}$ 5.0. ${ }^{40}$ At this low $\mathrm{pH}$, uric acid is 13 times less soluble than it is at a $\mathrm{pH}$ of 7.0 , and deposition of uric acid may lead to obstructive uropathy (Table 2) ${ }^{41-43}$ At uric acid levels of 594.8$892.2 \mu \mathrm{mol} / \mathrm{l}(10-15 \mathrm{mg} / \mathrm{dl})$, lethargy, nausea, and vomiting can occur, and signs of renal failure, such as hypertension and altered sensorium, may develop at uric acid levels above $1,189.6 \mu \mathrm{mol} / \mathrm{l}$ $(20 \mathrm{mg} / \mathrm{dl})$. Occasionally, TLS is accompanied by a coagulopathy. The fractional excretion of uric acid can be calculated as the ratio between uric acid and creatinine clearances. The fractional excretion is influenced by the extracellular volume, in that expansion increases whereas contraction decreases the urate excretion. This process is independent of urine flow rate. ${ }^{44}$

\section{Acute renal failure}

The causes of ARF are multifactorial, and complications can result in multiple organ failure and death. ${ }^{45}$ Phosphate and uric acid precipitation is the principal cause of renal insufficiency associated with TLS, and can subsequently lead to renal calculi and ARF and metabolic acidosis (Figure 1). ARF is defined as a sudden loss of renal function (over several hours to several days), and perturbs 
extracellular fluid balance, acid-base balance, electrolytes levels, and divalent cation regulation. An increased serum creatinine concentration, accumulation of other nitrogenous waste products, and often a decline in urinary output, are the hallmarks of this condition. ${ }^{46}$ Other endorgan dysfunction can also ensue, such as acute respiratory distress syndrome.

Determination of serum electrolytes offers valuable information, such as potassium levels, clues about volume status (e.g. high bicarbonate, elevated blood urea nitrogen:creatinine ratio, hypernatremia), acidosis, and anion gap (Box 1). Arguably, the most important laboratory test for a patient with ARF is urinanalysis. Both the urinary sediment and urinary indices in combination with serum values can often be extremely helpful in determining the cause of ARF. These indices are designed to determine whether tubular function is intact. A low sodium fractional excretion $(<1 \%)$ suggests that oliguria (and perhaps azotemia) is likely because of decreased renal perfusion, causing a decreased excretion of filtered sodium in the nephron, in an attempt to improve plasma volume and perfusion.

\section{CLASSIFICATION OF TUMOR LYSIS SYNDROME}

Hande and Garrow attempted to qualify the clinical and pathological characteristics of patients at risk of TLS. ${ }^{12}$ They classified TLS as either laboratory TLS or clinical TLS. This classification has several limitations. The laboratory TLS requires a $25 \%$ increase in the baseline laboratory values and development of TLS beyond 4 days of therapy, which include a 25\% increase in the serum phosphate, potassium, uric acid, or urea nitrogen concentrations, or a $25 \%$ decline in the serum calcium concentration. Clinical TLS requires one or more clinical manifestations (i.e. renal, neurological, or cardiac). Recently, Cairo and Bishop developed a new version of this classification, which incorporates laboratory and clinical TLS. ${ }^{47}$ In this new classification, clinical TLS requires one or more clinical manifestations along with criteria for laboratory TLS. The clinical TLS manifestation (i.e. renal, neurological, or cardiac) defines four grades of TLS.

\section{TREATMENT}

TLS requires prompt diagnosis and staging before cancer treatment can be initiated. TLS can either be present prior to chemotherapy or develop during treatment. To prevent TLS, it is
Table 1 Clinical consequence of metabolic disturbances.

\begin{tabular}{ll}
\hline Clinical features & Clinical consequences \\
\hline Hyperkalemia & $\begin{array}{l}\text { Cardiac: arrhythmias, ventricular tachycardia, } \\
\text { fibrillation, asystole, cardiac arrest } \\
\text { Neuromuscular: weakness, paresthesias, muscle } \\
\text { cramps, ascending flaccid paralysis } \\
\text { Others: nausea, vomiting, diarrhea, anorexia }\end{array}$ \\
& $\begin{array}{l}\text { Renal precipitation: urine decreases or stop, rise } \\
\text { in blood urea nitrogen and creatinine levels } \\
\text { Hyperphosphatemia }\end{array}$ \\
& $\begin{array}{l}\text { Coft tissue precipitation } \\
\text { Hypocalcemia }\end{array}$ \\
& $\begin{array}{l}\text { Neurovascular: hypotension, ventricular arrhythmias, } \\
\text { carpopedal spasms, paresthesias, laryngospasm } \\
\text { Mental status: confusion, delirium, hallucinations, } \\
\text { impaired memory, seizures }\end{array}$ \\
& $\begin{array}{l}\text { Renal: nausea, vomiting, oliguria, anuria, anorexia, } \\
\text { hematuria, possible rise in blood urea nitrogen and } \\
\text { creatinine levels, edema }\end{array}$ \\
\hline Hyperuricemia &
\end{tabular}

Table 2 Solubility of purine analogs and calcium phosphate at pH 5.0 and 7.0.

\begin{tabular}{lll}
\hline Metabolite & pH 5.0 & pH 7.0 \\
\hline Uric acid $(\mathrm{mg} / \mathrm{l})$ & 150 & 2,000 \\
Xanthine $(\mathrm{mg} / \mathrm{l})$ & 50 & 130 \\
Hypoxanthine $(\mathrm{mg} / \mathrm{l})$ & 1,400 & 1,500 \\
Calcium phosphate $(\mathrm{mg} / \mathrm{l})$ & 104.47 & 16.55 \\
\hline
\end{tabular}

important to identify high-risk patients, such as those with a large tumor load in both rapidly growing lymphoproliferative malignancies and solid tumors with extensive metastases, and those with a rapid rise in serum lactate dehydrogenase levels (Box 2). Tumor and drug characteristics, including the drug dose and density, should be taken into account (Box 3). Patients with TLS or who are at high risk of developing TLS should be evaluated with frequent clinical laboratory testing. In addition, it is necessary to monitor carefully the patient's fluid intake and output, weight and blood pressure at frequent intervals. Before cytotoxic chemotherapy begins, metabolic stability should be achieved, using methods such as those described below and summarized in Table 3 .

\section{Prophylaxis against tumor lysis syndrome}

Conventional management to prevent ARF caused by TLS consists of aggressive intravenous hydration (which should commence 3 days before chemotherapy), diuretic therapy, urinary alkalization, inhibition of urate production by high-dose allopurinol (400-600 mg/day) initiated 2 days before chemotherapy, uric acid oxidase administration and the gradual introduction of chemotherapy. ${ }^{45}$ 
www.nature.com/clinicalpractice/onc

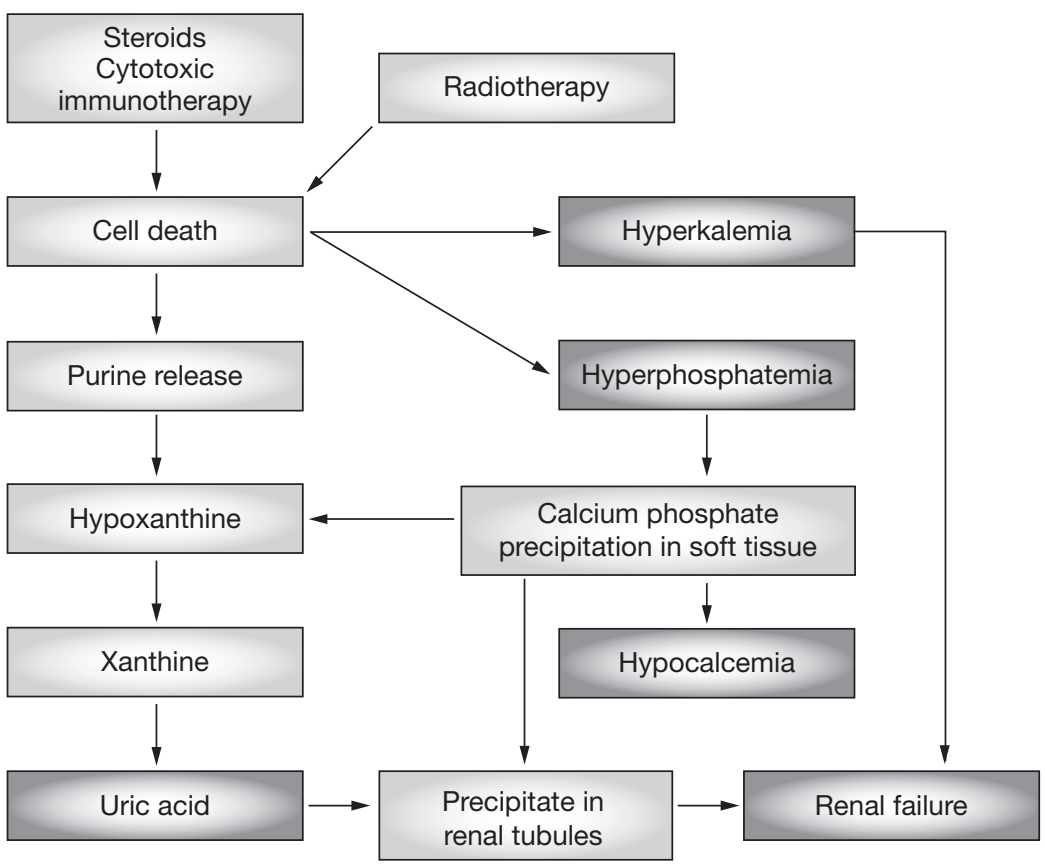

Figure 1 Metabolic imbalances due to TLS. Hyperuricemia, hyperkalemia, and hyperphosphatemia result from rapid destruction of malignant cells and release of intracellular ions, nucleic acids, proteins and their metabolites leading to ARF.

Table 3 Tumor lysis syndrome management.

\begin{tabular}{|c|c|}
\hline Treatment & Indication \\
\hline $\begin{array}{l}\text { Aggressive } \\
\text { hydration }\end{array}$ & $\begin{array}{l}\text { Prior to cytotoxic therapy ( } 24 \mathrm{~h} \text { before starting } \\
\text { chemotherapy) } \\
\text { Through the duration of treatment }\end{array}$ \\
\hline $\begin{array}{l}\text { Therapy of } \\
\text { hyperkalemia }\end{array}$ & $\begin{array}{l}\text { Cation exchange resins binding potassium (such as sodium } \\
\text { polystyrene sulfonate infused [10-30 ml] over } 1-5 \mathrm{~min} \text { ) } \\
\text { Calcium gluconate } \\
\text { Sodium bicarbonate to correct acidosis } \\
\text { Hypertonic dextrose and insulin } \\
\text { Diuretics } \\
\text { Inhaled } \beta \text {-agonist } \\
\text { Dialysis }\end{array}$ \\
\hline $\begin{array}{l}\text { Therapy of } \\
\text { hyperphosphatemia } \\
\text { and hypocalcemia }\end{array}$ & $\begin{array}{l}\text { Oral phosphate binders such as aluminum hydroxide } \\
\text { or aluminum carbonate } 30 \mathrm{ml} \text { four times daily } \\
\text { Hypertonic dextrose and insulin } \\
\text { Calcium gluconate (reserved for treating neuromuscolar } \\
\text { irritability) } \\
\text { Dialysis }\end{array}$ \\
\hline $\begin{array}{l}\text { Therapy of } \\
\text { hyperuricemia }\end{array}$ & $\begin{array}{l}\text { Sodium bicarbonate to alkalize urine }(75-100 \mathrm{mEq} / \mathrm{l}) \text {; } \\
\text { maintain urine } \mathrm{pH}>7.0 \\
\text { Allopurinol }\left(600-800 \mathrm{~g} / \mathrm{m}^{2} / \text { day orally for adults; }\right. \\
300 \mathrm{mg} / \mathrm{m}^{2} / \text { day orally for children) } \\
\text { Urate oxidase } \\
\text { Dialysis }\end{array}$ \\
\hline
\end{tabular}

In patients who are at high risk of developing TLS, preventive measures such as vigorous hydration and uricolytic agent must be instituted, in order to obtain an increased diuresis. Diuretic therapy and urinary alkalization and hydration should also be given immediately after chemotherapy.
Intravenous hydration

Excluding patients who are at risk of volume overload, such as elderly patients or those with heart failure, aggressive fluid administration has been recommended in patients at risk of TLS. ${ }^{2,48,49}$ Intravenous hydration should begin 2 days before chemotherapy and continue until 2-3 days after completion of treatment. Aggressive intravenous hydration increases intravascular volume and helps correct electrolyte disturbances by diluting extracellular fluid, thereby reducing serum potassium, uric acid and phosphate concentration. Increased intravascular volume via hydration also enhances renal blood flow, glomerular filtration rate and urinary volume, thereby decreasing the solute concentration in the distal nephron and medullary microcirculation, and possibly avoiding the need for dialysis (Figure 2). ${ }^{46,47,50}$ Patients should receive $2-3$ times the maintenance fluid volume administered as 5\% dextrose in a $0.2 \%$ sodium chloride solution. ${ }^{51}$

\section{Diuretic therapy}

Diuresis may be assisted with mannitol, furosemide or acetazolamide if adequate fluids are administered. Mannitol induces osmotic diuresis and is used when hydration alone is insufficient to maintain adequate diuresis. Diuretics can facilitate the renal excretion of potassium, but furosemide is not very effective if the renal tubules are affected by urate precipitation. In these instances, mannitol can be used. To induce alkaline diuresis, furosemide or acetazolamide can be used. To avoid a further increase of this electrolyte, it is important that potassium is not added to intravenous fluids. Diuretic therapy is not recommended unless volume overload from overhydration is present, which would necessitate temporary termination of intravenous fluids, and would also help avoid hyperkalemia.

\section{Urinary alkalization}

To reduce the risk of uric acid crystallization, it is important to increase the urinary $\mathrm{pH}$ to prevent uric acid nephropathy. The $\mathrm{pH}$ alkalization can be obtained by adding sodium bicarbonate to a hydrating physiologic solution, via oral administration of bicarbonate tablets, or by giving oral acetazolamide, but these approaches are only successful in patients without risk of systemic acidosis. ${ }^{52}$ Isotonic $0.45 \%$ sodium bicarbonate in normal saline with $5 \%$ dextrose should be infused at a rate of $150-300 \mathrm{ml} / \mathrm{h}$ to achieve effective diuresis and urinary alkalization at a 


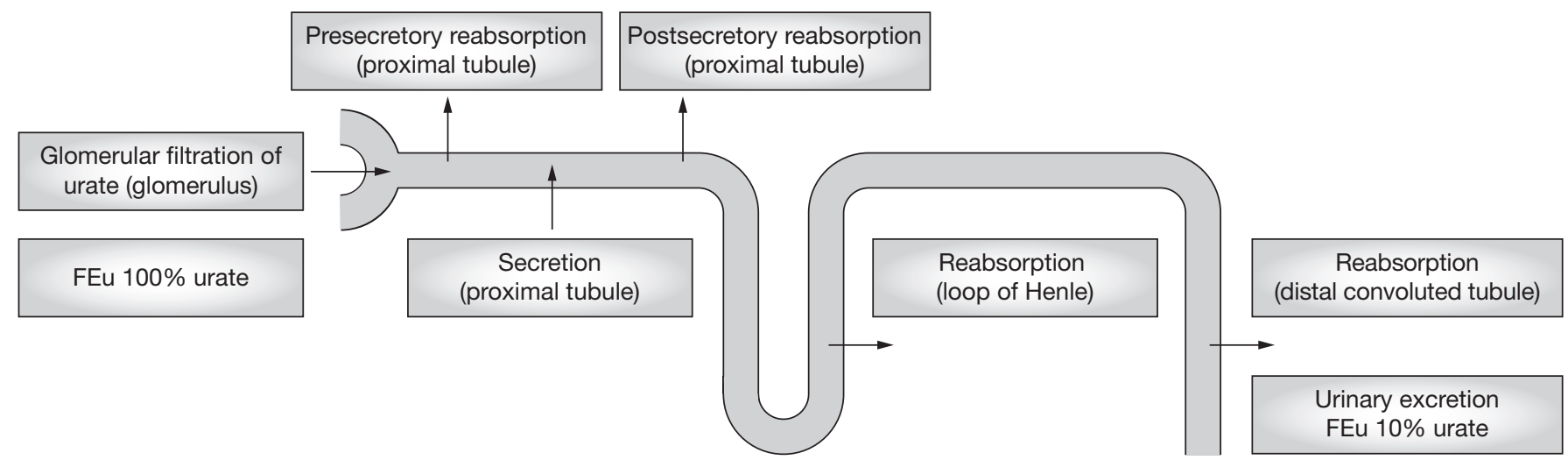

Figure 2 Uric acid load at various part of the kidney in the normal and diseased states. In patients with gout an increase of serum level and a decrease of urate transport in the nephron is observed. Uric acid of both normal patients and those with gout is filtered freely at the glomerulus and nearly all of this acid is reabsorbed before entering the distal convoluted tubule. The majority of uric acid in the urine is the result of secretion. Secretion and post-secretory reabsorption are thought to occur in the proximal tubule. Abbreviation: FEu, fractional excretion of urate (renal clearance of urate/glomerular filtration rate).

$\mathrm{pH}$ of $7.0-7.3,{ }^{53}$ permitting efficient excretion of uric acid in soluble form because of uric acid's increased solubility at this $\mathrm{pH}$.

The ways to achieve urinary alkalization are controversial. Conger and Falk reported that alkalization does not improve the abnormalities induced by hyperuricemia. ${ }^{54}$ The urinary alkalization might increase precipitation of calcium phosphate in the renal microvasculature and tubules, especially in patients with hyperphosphatemia (Table 3). ${ }^{55}$ This increase leads to obstructive nephropathy and a further decrease in glomerular filtration rate, exacerbating the risk of ARF. ${ }^{56}$ Systemic alkalization with sodium bicarbonate strengthens calcium-phosphate bonding and also reduces the concentration of ionized calcium, inducing a hypocalcemic state. Moreover, urinary alkalization may decrease the solubility of xanthine and increase the risk of xanthine nephropathy. If alkalosis occurs, bicarbonate should be stopped and the patient managed according to the degree of alkalosis present. A $0.9 \%$ sodium chloride solution can be injected intravenously, and potassium chloride can be administered if there is hypokalemia. Severe alkalosis can be accompanied by hyperirritability or tetany, and these symptoms can be controlled by calcium gluconate. An acidifying agent such as ammonium chloride might also be indicated in severe alkalosis.

\section{Hyperuricemia treatment with allopurinol and urate oxidase}

Allopurinol is a synthetic structural analog of hypoxanthine and competitively inhibits xanthine oxidase, thereby blocking the conversion of hypoxanthine and xanthine into uric acid. Since 1966, allopurinol combined with alkaline hydration has represented the standard treatment for malignancyassociated hyperuricemia, ${ }^{57}$ and allopurinol is also used in the management of patients at risk for TLS. Allopurinol administration reduces renal uric acid load, preventing its further production, but it does not affect existing uric acid. ${ }^{58}$

Allopurinol has a half-life of 60-180 minutes; the active metabolite of this agent, oxypurinol, is also an effective inhibitor of xanthine oxidase and remains active for $18-30$ hours. Since oxypurinol is cleared in the kidney, its half-life can be prolonged in patients with renal insufficiency. Allopurinol can be administered intravenously at a recommended dose of $200-400 \mathrm{mg} / \mathrm{m}^{2} /$ day. Skin rashes and hypersensitivity are the most frequent side effects of allopurinol, which is a less potent inhibitor of xanthine than oxypurinol, and in rare instances these symptoms can be life threatening. ${ }^{59}$ If allergic side effects occur, allopurinol treatment should be discontinued immediately. Because high uric acid can exist before allopurinol treatment is initiated, a period of 2-3 days is generally necessary for uric acid levels to decrease after initiation of allopurinol treatment.

Although allopurinol is effective in the reduction of hyperuricemia, it has some disadvantages, including the need to delay chemotherapy, unfavorable drug interactions, the need for dose reduction in patients with impaired renal function, allergic side effects, and induction of xanthine nephropathy. ${ }^{60,61}$ Allopurinol does not directly alter the level of circulating uric acid, and 
nephropathy can develop because of renal overload of the uric acid precursor. If cellular lysis persists, xanthine and hypoxanthine cannot be converted into uric acid, and high quantities of these agents enter the renal tubules, where they can crystallize and produce xanthine nephropathy ${ }^{60}$ Xanthine has a lower solubility than uric acid, and there have been case reports of xanthine stone formation. ${ }^{62,63}$ It is necessary to maintain neutral or slightly alkaline urinary $\mathrm{pH}$ and urinary excretion of 2 liters/day at least in adult patients, to avoid xanthine crystalluria.

Urate oxidase (also known as uricase), a proteolytic enzyme not present in humans and other primates, oxidizes uric acid in water-soluble allantoin. Since allantoin is readily water soluble, its elimination by urinary excretion is less problematic than seen with xanthine or uric acid. In fact, the kidneys readily excrete allantoin, and its reactive by-product, hydrogen peroxide, is neutralized by catalase to oxygen and water. Hydrogen peroxide produced as a result of uric acid breakdown, caused kidney complications such as ARF in patients with leukemia and lymphoma. ${ }^{64}$ Non-recombinant urate oxidase derived from cultures of Aspergillus flavus has been used in Europe since 1974 as a short-term adjunct to cytolytic therapy for childhood leukemia and other myeloproliferative and lymphoproliferative disorders. 2,61,65 The use of non-recombinant urate oxidase is limited by potential immunogenicity and declining efficacy caused by production of antiuricase antibodies, and side effects such as allergic rash, anaphylaxis, and bronchospasm. ${ }^{66}$ Recently, the recombinant urate oxidase rasburicase (Fasturtec ${ }^{\circledR}$; Sanofi-Aventis, Paris, France) was developed to overcome these problems associated with the non-recombinant product.

The primary advantage of recombinant urate oxidase is its rapid onset of action. Urate oxidase has been used in the management of patients at risk for TLS; ${ }^{66-72}$ the drug is used widely to treat TLS in Europe, and was approved for this purpose by the US FDA in 2002. Urate oxidase can rapidly decrease serum levels of uric acid with associated diuresis. The efficacy and safety of rasburicase is currently being tested and compared with that of allopurinol in a phase III clinical trial. ${ }^{65}$ The interim results showed that rasburicase induced a decrease in uric acid concentration in all pediatric and adult patients who received this treatment, and this therapy appears to be well tolerated. ${ }^{68}$ Rasburicase has some advantages over allopurinol in that it does not require alkalization, meaning that phosphorus excretion is facilitated. Moreover, a retrospective comparison of rasburicase and allopurinol showed that the former was significantly more effective at reducing uric acid levels, and also improved renal function as demonstrated by lower blood urea nitrogen and creatinine levels. ${ }^{61,67}$

The pegylated form of urate oxidase, PEGuricase, reduces plasma uric acid levels without antigenicity. Two groups detected antibodies to rasburicase in 17 of 121 patients and in 7 of 97 patients using an enzyme-linked immunosorbent assay. ${ }^{61}$ The detection of antibodies to the pegylated urate oxidase was not associated with the clinical status of the patients or the occurrence of adverse events; this agent also has a longer duration of action than unmodified urate oxidase without an adverse reaction. ${ }^{73,74}$ Several data suggest that rasburicase is effective and well tolerated in the prevention and treatment of chemotherapy-induced hyperuricemia, whereas hypersensitivity reactions were observed in nearly $5 \%$ of patients treated with non-recombinant urate oxidase. ${ }^{65}$ The first studies to demonstrate that the efficacy of recombinant urate oxidase is superior to that of allopurinol was in childhood leukemia. ${ }^{65,75}$ Guidelines for the use of rasburicase are still being developed, but owing to the agent's high cost there is controversy surrounding its usage. A significant advantage of rasburicase is that chemotherapy can be promptly initiated with a significant reduction in the risk of renal dysfunction requiring dialysis. It would seem reasonable to use this drug to treat patients who are at high risk of TLS but cannot ingest oral allopurinol or are allergic to this drug, which accounts for approximately $2 \%$ of patients treated with allopurinol, particularly elderly patients with renal insufficiency. ${ }^{76,77}$ Rasburicase is also indicated in patients who present with hyperuricemia, high tumor burden, or renal problems. Although rasburicase prevents renal uric acid accumulation, it causes phosphate reabsorption, and calcium phosphate deposition remains a concern. Rasburicase may also be used to spare the costs and complications of cytoreductive procedures. ${ }^{74}$

\section{Hyperkalemia treatment}

Treatment options of hyperkalemia are directed primarily at counteracting the effect on myocardium, stabilizing cardiac membrane (calcium gluconate) and preventing lifethreatening cardiac arrhythmias; another intervention (glucose with insulin, sodium bicarbonate, 
inhaled $\beta$-agonist, potassium-binding resins, and dialysis) is aimed at reducing the potassium level. Moderate hyperkalemia can be treated with Kayexalate ${ }^{\circledR}$ (sodium polystyrene sulfonate; Sanofi Pharmaceuticals, Inc., New York, NY), but the effects of this agent are not immediate, partly because it functions as an ion-exchange resin in the gastrointestinal tract. ${ }^{49}$ Recent data have demonstrated colonic necrosis associated with the use of sodium polystyrene sulfonate in critically ill and unstable patients; ${ }^{78}$ therefore, use of this agent is not indicated for the treatment of acute toxicities, such as life-threatening arrhythmias. For severe cardiac toxicities, calcium gluconate may be used; membrane stabilization is achieved with $10 \%$ calcium gluconate to prevent life-threatening cardiac arrhythmias. ${ }^{79,80}$ While cardiac protection may be immediate, the effects are transient and should be used only in patients with significant electrocardiographic changes. Other treatment modalities for severe hyperkalemia with or without electrocardiographic changes include hypertonic glucose and insulin, loop diuretics, and bicarbonate. There is evidence that bicarbonate will not result in lowering of serum potassium unless there is a severe metabolic acidosis. ${ }^{45}$

\section{Hyperphosphatemia and hypocalcemia treatment}

Treatment of hyperphosphatemia should take into consideration the underlying biological alterations (e.g. phosphorus levels $>1.62 \mathrm{mmol} / \mathrm{l}$ [ $>5 \mathrm{mg} / \mathrm{dl}]$ that suggest the need for medical intervention. A mild asymptomatic hyperphosphatemia in patients with chronic renal failure can be corrected with a reduced dietary phosphate intake; phosphate binders such as aluminum hydroxide or aluminum carbonate ( $30 \mathrm{ml}$ taken four times daily) will decrease the absorption of phosphate in the gout. ${ }^{49}$ The longterm use of aluminum compounds is not recommended, especially in patients with advanced chronic kidney disease, because aluminum is toxic. A severe hyperphosphatemia in patients with end-stage renal failure can be corrected by hemodialysis or peritoneal dialysis. Usually, treatment of hyperphosphatemia will also correct any related hypocalcemia. ${ }^{80}$ Calcium itself should not be administered as it might precipitate metastatic calcifications. ${ }^{81}$ Treatment of hypocalcemia consists of parenteral calcium (e.g. calcium gluconate) administered intravenously over 10 minutes. If calcium gluconate is injected, however, severe arrhythmias can occur. When recurrent hypocalcemia is present, a continuous intravenous infusion of calcium gluconate can be initiated.

Acute renal failure treatment

Although the biochemical and clinical abnormalities associated with TLS are usually reversible when recognized and treated appropriately, studies showed that $40 \%$ of patients died during the acute episode of TLS. ${ }^{12,21,82}$ Intravascular volume expansion (i.e. 3 liters $/ \mathrm{m}^{2} /$ day) with hydration and forced diuresis has played a critical prophylactic role in the prevention of ARF. Despite these prophylactic efforts, hemodialysis should be considered for every patient with excessively elevated uric acid, phosphate and/or potassium, and in patients with ARF to control volume and manage uremia. Data comparing the various dialytic modalities (hemodialysis, peritoneal dialysis and continuous dialysis hemofiltration) are lacking. Furthermore, initiation of hemodialysis before severe pathophysiologic deterioration occurs has been suggested to benefit patients with multiorgan failure and ARF. ${ }^{83}$ In general, hemodialysis is the preferred treatment, as it can rapidly correct any life-threatening electrolyte changes. Dialysis should be performed every 12 hours until renal/hepatic function and urinary volume are restored. Dialysis every 12-24 hours may also be necessary in patients presenting with a large phosphate burden. Peritoneal dialysis is much less efficient than hemodialysis in correcting metabolic abnormalities, and hemodialysis affords much higher clearance of both uric acid and phosphorus. ${ }^{7,84,85}$

\section{CONCLUSION}

TLS is an oncologic emergency, and as moreaggressive and more-highly-targeted therapies, high doses of cytotoxic agents and biologic response modifiers have become a standard therapy, TLS will be more frequently encountered. ${ }^{85}$ The recognition and treatment of TLS arising in patients with cancer is very important, and clinicians should not only treat the metabolic abnormalities of the acute TLS and its complications, but, for preventive measures to be put in place, identify patients at risk as early as possible. The potential for TLS in patients with bulky treatment-sensitive tumors who present with renal insufficiency, dehydration, hyperuricemia and elevated serum lactate dehydrogenase levels must be recognized, to allow for appropriate monitoring after treatment and early initiation 
of therapeutic measures if necessary. As advances are made in the treatment of patients with these common tumors, it will become increasingly important for physicians to recognize the risk factors and biochemical abnormalities associated with TLS. ${ }^{21}$

\section{KEY POINTS}

- The rapid and massive destruction of tumor cells caused by cancer therapy can lead to release of cellular breakdown products

- The characteristic signs of TLS are hyperuricemia, hyperphosphatemia, hyperkalemia and hypocalcemia

- Aggressive intravenous hydration, diuretic therapy, $\mathrm{pH}$ alkalization and the correction of electrolytic balance are the basis of prophylaxis therapy

- Rasburicase, a recombinant urate oxidase prevents renal uric acid accumulation and plays a prophylactic role in ARF

\section{References}

1 Castro MP et al. (1999) Acute tumor lysis syndrome associated with concurrent biochemotherapy of metastatic melanoma: a case report and review of the literature. Cancer 85: 1055

2 Jones DP et al. (1995) Tumor lysis syndrome: pathogenesis and management. Pediatr Nephrol 9: 206-212

3 Co CS et al. (1983) Urinary uric acid excretion in patients with carcinoma of the cervix undergoing intracavitary radium therapy. Radiat Med 1: 245-247

4 Jasek AM and Day HJ (1994) Acute spontaneous tumor lysis syndrome. Am J Hematol 47: 129-131

5 Alkhuja $\mathrm{S}$ and Ulrich $\mathrm{H}$ (2002) Acute renal failure from spontaneous acute tumor lysis syndrome: a case report and review. Ren Fail 24: 227-232

6 Feld J et al. (2000) Acute spontaneous tumor lysis syndrome in adenocarcinoma of the lung: case report. Am J Clin Oncol 23: 491-493

7 Agha-Razii M et al. (2000) Continuous veno-venous hemodiafiltration for the treatment of spontaneous tumor lysis syndrome complicated by acute renal failure and severe hyperuricemia. Clin Nephrol 54: 59-63

8 Salkowsky F (1870) Beitrage Zur Kenntins der Leukamie. Virchows Arch Pathol Anat 50: 174-177

9 Bedrna J et al. (1929) harnleiterverschluss nach Bestrahlung chronischer leukämien mit röntgenstrahlen. Med Klin 25: 1700-1701

10 Crittenden DR and Ackerman GL (1977) Hyperuricemic acute renal failure in disseminated carcinoma. Arch Intern Med 137: 97-99

11 Cohen LF et al. (1980) Acute tumor lysis syndrome: a review of 37 patients with Burkitt's lymphoma. Am J Med 68: 486-491

12 Hande KR and Garrow GC (1993) Acute tumor lysis syndrome in patients with high grade non-Hodgkin lymphoma. Am J Med 94: 133-139

13 Tsokos GC et al. (1981) Renal and metabolic complications of undifferentiated and lymphoblastic lymphomas. Medicine (Baltimore) 60: 218-229

14 Montalban C et al. (1994) Tumor lysis syndrome after treatment of chronic lymphocitic leukaemia with fludarabine. Postgrad Med J 70: 651-652
15 Trendle MC and Tefferi A (1994) Tumor lysis syndrome after treatment of chronic lymphocytic leukemia with cladribine. N Engl J Med 330: 1090

16 Dann EJ et al. (1993) Brief report: tumor lysis syndrome following treatment with 2-chlorodeoxyadenosine for refractory chronic lymphocitic leukaemia. $N$ Engl $J$ Med 329: 1547-1548

17 Mulligan SP and Dean MG (1994) Fludarabine causing tumor lysis syndrome in chronic lymphocitic leukaemia. Aust NZJ Med 24: 406-407

18 Drakos $\mathrm{P}$ et al. (1994) Tumor lysis syndrome in nonhematologic malignancies: report of a case and review of the literature. Am J Clin Oncol 17: 502-505

19 Cech P et al. (1986) Tumor lysis syndrome after tamoxifen flare. N Engl J Med 315: 263-264

20 Ustundag Y et al. (1997) Acute tumor lysis syndrome associated with paclitaxel. Ann Pharmacother 31: 1548-1549

21 Kalemkerian GP et al. (1997) Tumor lysis syndrome in small cell carcinoma and other solid tumors. $A m \mathrm{~J}$ Med 103: 363-367

22 Vogelzang NJ et al. (1983) Tumor lysis syndrome after induction chemotherapy of small-cell bronchogenic carcinoma. JAMA 249: 513-514

23 Hussein AM and Feun LG (1990) Tumor lysis syndrome after induction chemotherapy in small-cell lung carcinoma. Am J Clin Oncol 13: 10-13

24 Persons DA et al. (1998) Tumor lysis syndrome and acute renal failure after treatment of non-small cell lung carcinoma with combination irinotecan and cisplatin. Am J Clin Oncol 21: 426-429

25 Barton JC (1989) Tumor lysis syndrome in nonhematopoietic neoplasms. Cancer 64: 738-740

26 Yokoi Ket al. (1997) Tumor lysis syndrome in invasive thymoma with peripheral blood T-cell lymphocytosis. Am J Clin Oncol 20: 86-89

27 Tomlinson GC and Solberg LA Jr (1984) Acute tumor lysis syndrome with metastatic medulloblastoma: a case report. Cancer 53: 1783-1785

28 Bilgrami SF and Fallon BG (1993) Tumor lysis syndrome after combination chemotherapy for ovarian cancer. Med Pediatr Oncol 21: 521-524

29 Khan J and Broadbent VA (1993) Tumor lysis syndrome complicating treatment of widespread metastatic abdominal rhabdomyosarcoma. Pediatr Hematol Oncol 10: 151-155

30 Khalil A et al. (1998) Fatal acute tumor lysis syndrome following treatment of vulvar carcinoma: case report. Eur J Gynaecol Oncol 19: 415-416

31 Dirix LY et al. (1991) Tumor lysis syndrome in a patient with metastatic Merkel cell carcinoma. Cancer 67: 2207-2210

32 Wossmann W et al. (2003) Incidence of tumor lysis syndrome in children with advanced stage Burkitt's lymphoma/leukaemia before and after introduction of prophylactic use of urate oxidase. Ann Hematol 82: 160-165

33 Baeksgaard L and Sørensen JB (2003) Acute tumor lysis syndrome in solid tumors-a case report and review of the literature. Cancer Chemother Pharmacol 51: 187-192

34 Hediger MA et al. (2005) Molecular physiology of urate transport. Physiology (Bethesda) 20: 125-133

35 Flombaum CD (2000) Metabolic emergencies in the cancer patient. Semin Oncol 27: 322-334

36 Mattu A et al. (2000) Electrocardiographic manifestations of hyperkalemia. Am J Emerg Med 18: 721-729

37 Shiber JR and Mattu A (2003) Serum phosphate abnormalities in the emergency department. J Emerg Med 23: 395-400

38 Jean-Jacques Body and Roger Bouillon (2003) Emergencies of calcium homeostasis. Rev Endocr Metab Disorders 4: 167-175 
39 Duncan H and Dixon AS (1960) Gout, familial hypericaemia, and renal disease. Q J Med 29: 127-135

40 Wilcox WR et al. (1972) Solubility of uric acid and monosodium urate. Med Biol Eng 10: 522-531

41 Klinenberg JR et al. (1975) Hyperuricemic nephropathy: pathologic features and factors influencing urate deposition. Nephron 14: 88-98

42 Conger JD and Falk SA (1977) Intrarenal dynamics in the pathogenesis and prevention of acute urate nephropathy. J Clin Invest 59: 786-793

43 Rieselbach RE et al. (1964) Uric acid excretion and renal function in the acute hyperuricemia of leukaemia: pathogenesis and therapy of uric acid nephropathy. Am J Med 37: 872-884

44 Kelton J et al. (1978) A rapid method for the diagnosis of acute uric acid nephropathy. Arch Int Med 138: 612-615

45 Van Der Klooster JM et al. (2000) Asystole during combination chemotherapy for non-Hodgkin's lymphoma: the acute tumor lysis syndrome. Neth $\mathrm{J}$ Med 56: 147-152

46 Albright RC Jr (2001) Acute renal failure: a practical update. Mayo Clin Proc 76: 67-74

47 Cairo MS and Bishop M (2004) Tumor lysis syndrome: new therapeutic strategies and classification. $\mathrm{Br} \mathrm{J}$ Haematol 127: 3-11

48 Lorigan PC et al. (1996) Tumour lysis syndrome, case report and review of the literature. Ann Oncol 7: 631-636

49 Sallan S (2001) Management of acute tumor lysis syndrome. Semin Oncol 28 (Suppl 5): S9-S12

50 Sewani H and Rabatin JT (2002) Acute tumor lysis syndrome in a patient with mixed small cell and non-small cell tumor. Mayo Clin Proc 77: 722-728

51 Kingston ME (1974) Methods of rehydration. J Trop Pediatr Environ Child Health 20: 55-56

52 Lynch RE et al. (1997) Renal and metabolic complications of childhood non-Hodgkin's lymphoma. Semin Oncol 4: 325-334

53 Arrambide K and Toto RD (1993) Tumor lysis syndrome. Semin Nephrol 13: 273-280

54 Conger JD and Falk SA (1997) Intrarenal dynamics in the pathogenesis and prevention of acute urate nephropathy. J Clin Invest 59: 786-793

55 Massry SG et al. (1973) Excretion of phosphate and calcium: physiology of their renal handling and relation to clinical medicine. Arch Intern Med 131: 828-859

56 Monballyu J et al. (1984) Transient acute renal failure due to tumor-lysis-induced severe phosphate load in a patient with Burkitt's lymphoma. Clin Nephrol 22: 47-50

57 DeConti RC and Calabresi P (1966) Use of allopurinol for prevention and control of hyperuricemia in patients with neoplastic disease. N Engl J Med 274: 481-486

58 Hande KR et al. (1981) Postchemotherapy purine excretion in lymphoma patients receiving allopurinol. Cancer Res 41: 2273-2279

59 Earll JM and Saavedra M (1983) Oxipurinol therapy in allopurinol-allergic patients. Am Fam Physician 28: 147-148

60 Andreoli SP et al. (1986) Purine excretion during tumor lysis in children with acute lymphocytic leukemia receiving allopurinol: relationship to acute renal failure. J Pediatr 109: 292-298

61 Pui CH et al. (1997) Urate oxidase in prevention and treatment of hyperuricemia associated with lymphoid malignancies. Leukaemia 11: 1813-1816

62 Greene ML et al. (1969) Urinary xanthine stones-a rare complication of allopurinol therapy. N Engl J Med 280: 426-427

63 Band PR et al. (1970) Xanthine nephropathy in a patient with lymphosarcoma treated with allopurinol. N Engl J Med 283: 354-357
64 Goldman SC et al. (2001) A randomized comparison between rasburicase and allopurinol in children with lymphoma or leukemia at high risk for tumor lysis. Blood 97: 2998-3003

65 Pui CH et al. (2001) Recombinant urate oxidase for the prophylaxis or treatment of hyperuricemia in patients with leukemia or lymphoma. J Clin Oncol 19: 697-704

66 Leverger $\mathrm{G}$ and Patte C (2003) Therapeutic progress in acute lymphoblastic leukemias and stage IV Burkitt-type lymphomas in children. Bull Acad Natl Med 187: 743-755

67 Patte $\mathrm{C}$ et al. (2001) European experience in the treatment of hyperuricemia. Semin Hematol 38 (Suppl 10): S9-S12

68 Patte C et al. (2002) Urate-oxidase in the prevention and treatment of metabolic complications in patients with B-cell lymphoma and leukaemia, treated in the Societe Francaise d'Oncologie Pediatrique LMB89 protocol. Ann Oncol 13: 789-795

69 Leach KG et al. (1985) Serial measurement of renal plasma flow. Eur J Nucl Med 11: 33-35

70 Jankovic M et al. (1985) Urate-oxidase as hypouricemic agent in a case of acute tumor lysis syndrome. Am J Pediatr Hematol Oncol 7: 202-204

71 Masera G et al. (1982) Urate-oxidase prophylaxis of uric acid-induced renal damage in childhood leukemia. J Pediatr 100: 152-155

72 Navolanic PM et al. (2003) Elitek-rasburicase: an effective means to prevent and treat hyperuricemia associated with tumor lysis syndrome. Leukemia 17: 499-514

73 Ganson NJ et al. (2005) Control of hyperuricemia in subjects with refractory gout, and induction of antibody against poly(ethylene) glycol (PEG), in a phase I trial of subcutaneous PEGylated urate oxidase. Arthritis Res Ther 8: 1-10

74 Hammel M et al. (2003) Successful treatment of hyperuricemia with low dose of recombinant urate oxidase in four patients with haematologic malignancy and tumor lysis syndrome. Leukemia 17: 2542-2544

75 Mclnnes GT et al. (1981) Acute adverse reactions attributed to allopurinol in hospitalised patients. Ann Rheum Dis 40: 245-249

76 Arellano F and Sacristan JA (1993) Allopurinol hypersensitivity syndrome: a review. Ann Pharmacother 27: 337-343

77 Macfarlane RJ et al. (2004) Rasburicase prevents tumor lysis syndrome despite extreme hyperleukocytosis. Pediatr Nephrol 19: 924-927

78 Scott TR et al. (1993) Colonic necrosis following sodium polystyrene sulphonate (Kayexalate)-sorbitol enema in a renal transplant patient: report of a case and review of the literature. Dis Colon Rectum 36: 607-609

79 Altman A (2001) Acute tumor lysis syndrome. Semin Oncol 28 (Suppl 5): S3-S8

80 Jeha S (2001) Tumor Lysis Syndrome. Semin Hematol (Suppl) 10: S4-S8

81 Yarpuzlu AA (2003) A review of clinical and laboratory findings and treatment of tumor lysis syndrome. Clin Chim Acta 333: 1318

82 Hande KR and Garrow GC (1993) Acute tumor lysis syndrome in patients with high-grade non-Hodgkin's lymphoma. Am J Med 94: 133-139

83 Frisk P et al. (2002) Renal function after autologous bone marrow transplantation in children: a long term prospective study. Bone Marrow Transplant 29: 129-136

84 DuBose TD Jr et al. (1997) Acute renal failure in the 21st century: recommendations for management and outcomes assessment. Am J Kidney Dis 29: 793-799

85 Davidson MB et al. (2004) Pathophysiology, clinical consequences, and treatment of tumor lysis syndrome. Am J Med 116: 546-554

\section{Competing interests}

The authors declared they have no competing interests. 